\title{
On the Sustainability of Photochemical Reactions
}

\author{
Christian G. Bochet*
}

\begin{abstract}
Photochemical reactions are often presented as intrinsically environmentally friendly ('green'). This may be indeed the case, but only in particular circumstances. This short essay comments on various aspects of the greenness of photochemical reactions, both in a historical (when only mercury-based hard UV light sources were available) and a current (with the recent introduction of LED technology and photoredox catalysis) perspective.
\end{abstract}

Keywords: Green chemistry · Photochemistry

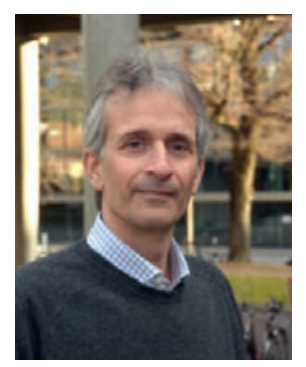

Christian Bochet got a MSc degree in 1991 in inorganic chemistry (Prof. Alan Williams) and a PhD in organic chemistry in 1996 (Prof. Wolfgang Oppolzer) at the University of Geneva. After a postdoctoral stay at Stanford University (Prof. Barry M. Trost), he set up an independent research group in organic photochemistry at the University of Geneva. In 2002, he was awarded a SNF-professorship and moved to the University of Fribourg, where he became full professor in 2006. He was dean of the Faculty of Science and Medicine from 2016 to 2019. His current interests include organic synthesis in general, total synthesis of (natural) products and organic photochemistry. When not doing chemistry, he enjoys playing violin in the Geneva Symphony Orchestra.

The possibility to harness sunlight to produce useful fine chemicals and fuel was recognized more than a century ago; it was nicely, if not lyrically, illustrated by Ciamician in his famous dream. ${ }^{[1]}$

"On the arid lands there will spring up industrial colonies without smoke and without smokestacks; forests of glass tubes will extend over the plains and glass buildings will rise everywhere; inside of these will take place the photochemical processes that hitherto have been the guarded secret of the plants, but that will have been mastered by human industry which will know how to make them bear even more abundant fruit than nature, for nature is not in a hurry and mankind is."

Since that time, many new photoinduced processes have been developed, and justified through this angle, sometimes abusively. The aim of this essay is to debunk a few myths and provocatively discuss on the real sustainable nature of photochemical reactions. This is not a unique endeavour, and several other similar discussions have occurred in the past, for photochemical reactions or others. ${ }^{[2]}$ We believe that this discussion is important, because there is a real danger that the whole field will be (again) forgotten by not being able to fully satisfy unreasonable expectations, and that the photo frenzy will follow the fate of bio, combi and perhaps even nano buzzwords fashion.

In fact, the evolution of the number of publications mentioning Green photochemistry (Fig. 1, blue line, right vertical axis) follows the trend seen in Green chemistry (Fig. 1, red line, left vertical axis). ${ }^{[3]}$ Interestingly, whereas Green chemistry starts from zero, which is reasonable since the initial publication dates from 1998, photochemistry was associated with greenness earlier, which is also understandable since it was one of the drivers of research in the field already in Ciamician's time. Since only photons as supplementary reagents are used, photochemical reactions are, most of the time, atom-economical. ${ }^{[4]}$

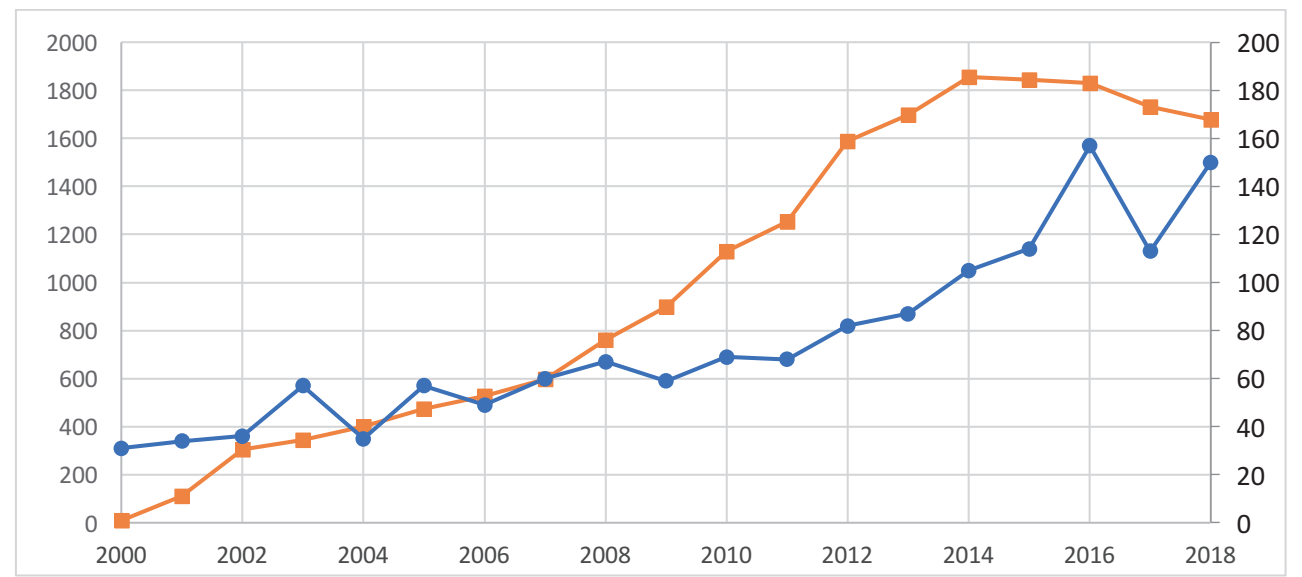

Fig. 1. Annual new publications on green chemistry (blue line, right scale) and green photochemistry (orange line, left scale). 
Let us first revisit the basics of Green Chemistry. Twelve principles were stated in the initial landmark publication: ${ }^{[5]}$

1. Prevention

2. Atom Economy

3. Less Hazardous Chemical Syntheses

4. Designing Safer Chemicals

5. Safer Solvents and Auxiliaries

6. Design for Energy Efficiency

7. Use of Renewable Feedstocks

8. Reduce Derivatives

9. Catalysis

10. Design for Degradation

11. Real-time Analysis for Pollution Prevention

12. Inherently Safer Chemistry for Accident Prevention

On top of that, another twelve principles of engineering were later proposed:[6,7]

1. Inherently non-hazardous and safe

2. Minimize material diversity

3. Prevention instead of treatment

4. Renewable material and energy inputs

5. Output-led design

6. Very simple

7. Efficient use of mass, energy space and time

8. Meet the need

9. Easy to separate by design

10. Networks for exchange of local mass and energy

11. Test the life cycle of the design

12. Sustainability throughout product life cycle

The use of a photon as a reagent is by essence very appealing, and it is very surprising, at least by considering only this aspect, that the general interest by the synthetic community faded after the initial Ciamician-induced spark, before coming again in fashion in the 1970s and fading again. The main drawbacks that were invoked were the poor predictability of the outcome (true: the fate of an excited state depends on many parameters, and thus it is more difficult to control the tuning conditions), ${ }^{\left[{ }^{[3}\right.}$ difficulty to generate high-quality UV light (true: mercury lamps were the only widespread UV sources, but the LED revolution, also in the UV spectral range, changed the situation in the last decade), engineering problems to deliver the light at the desired location (true, but again LED sources are much more easily mounted on reactors; on the other hand, quartz glassware is still necessary for wavelengths shorter than $350 \mathrm{~nm}$ ) and a limited catalogue of possible transformations (true at the beginning, improving with time). The last 10 years have shown a tremendous revival of photochemical reactions, by the realization that visible light could be harvested by a catalytic amount of a metal complex (e.g. $\left.\mathrm{Ru}(\mathrm{bpy})_{3} \mathrm{Cl}_{2}\right)$ and then exploited by a chemical reaction, such as a redox process. ${ }^{[9]}$ This strategy, photoredox catalysis, very rapidly changed the face of photochemistry: a multitude of reactions that were reserved to the cumbersome hard UV range became possible in the visible range (thus voiding most of the drawbacks mentioned above), and new reactions were (and still are being) discovered.

The ultimate sustainable photochemical process is the conversion of solar energy into chemical energy, which can then be, on demand, exploited under one or another form. Several types of reactions were studied in the 1970s. Among them, the most promising was the norbornene - quadricyclane [2+2] cycloaddition, which can then be reverted releasing thermal energy (Scheme 1). This reaction proceeds at $366 \mathrm{~nm}$, and can be repeated in a number of cycles before significant degradation. The stored enthalpy is $1.19[\mathrm{~kJ} / \mathrm{g}]$, with a quantum efficiency of $90 \%$, and a temperature of return between 135 and $200{ }^{\circ} \mathrm{C}$. This process was actually once considered as an energy storage, but overall profitability was shown to be unfavourable, and it was thus abandoned.[10] The cost of a $100 \mathrm{~kW}$ plant was evaluated at 2.4 Mio\$ (in 1981) for the infrastructure, plus 7.38 Mio\$ for the norbornadiene itself. In the best scenario (i.e. with an infinite number of cycles), the cost of steam produced in this way was 50 times higher than in a conventional way.

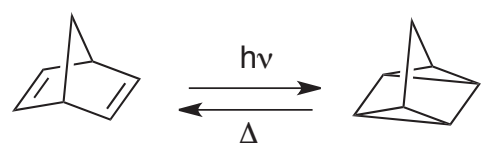

Scheme 1. The norbornadiene-quadricyclane photoisomerisation as chemical energy storage.

Another ideal photochemical reaction is the splitting of water into hydrogen and oxygen, of which the energy can be exploited either chemically or electrically. Such a reaction is possible, as shown by many publications, ${ }^{[11,12]}$ but still not economically on par with existing technologies: burning organic matter into carbon dioxide remains by far the most widely used chemical reaction, something that is precisely the opposite of what synthetic chemists are aiming for.

An inherently sustainable process would use photons that are cheap, abundant and renewable, and so the use of sunlight would seem a legitimate option. Indeed, significant efforts were made in this direction, for example the work on the PROPHIS reactor. ${ }^{[13]}$ This is a parabolic through collector (PTC), concentrating sunlight into glass tubes by a factor of $c a$. 15, installed in Cologne, Germany. With a surface of $24 \mathrm{~m}^{2}$, up to 38 moles of 300-400 nm photons were collected, together with 227 moles of 400-500 nm photons, for a sunshine duration of 12.75 hours. The unreliability of weather in our regions is clearly exemplified by the fact that the next day, the sunshine duration was just half an hour, and 4.25 hours the day after. That being said, these PTC modules are widely used throughout the world (mainly to produce steam), and so the engineering costs to reconvert them into photoreactors are not massive. All in all, weather permitting, more than 250 moles of a free and traceless reagent are not insignificant. Using this setup, a photo-Diels Alder reaction was carried out, as well as the photooxygenation of citronellol and 1,5-dihydroxynaphthalene (Scheme 2). ${ }^{[13,14]}$ A much simpler solar photoreactor was also built using readily available components in our own laboratory. ${ }^{[15]}$ Far from being the panacea, in particular under our latitudes, solar photochemistry may find its role in regions where sunshine is reliable and/or directly where raw materials are extracted.

A crucial aspect that should not be neglected is the overall simplification of a synthetic route by including a photochemical step, allowing connections between atoms that would not have been possible otherwise. A classic example is the total synthesis of Longifolene. The first successful synthesis of this sesquiterpene totalled 27 steps and an overall yield of $1.7 \%$ from the WielandMischler ketone (17 of these steps were necessary to access the latter!), a remarkable achievement at that time. ${ }^{[16]}$ By the clever use of a photochemical de Mayo reaction (a [2+2] cycloaddition/ retroaldol cascade), Oppolzer and Godel cut down the number of steps to 7 and improved the overall yield to $25 \%$ from cyclopentenecarboxylic acid. ${ }^{[17]}$ Since that time, many other illustrations of these simplifications have been published, such as the syntheses of Cedrene ${ }^{[18,19]}$ or Hirsutene, ${ }^{[20,21]}$ both polycyclic sesquiterpenes. The latter core was assembled by Mehta in only four steps in a $28 \%$ overall yield. ${ }^{[21]}$ Further examples are cited in the recent excellent review by Kärkäs, Porco and Stephenson. ${ }^{[22]}$ Thus, regardless of the greenness of the photochemical step itself (many of the above examples required a high-pressure mercury lamp, large amount of solvents, and a difficult separation of structurally similar isomers), the reduction in the number of steps is so high that it compensates many shortcomings. Therefore, the strategic 
<smiles>O=C1C=CC(=O)c2ccccc21</smiles><smiles>[R]C(=O)c1cc(O)c2ccc([13CH])cc2c1O</smiles><smiles>Oc1cccc2c(O)cccc12</smiles>

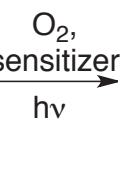<smiles>O=C1C=CC(=O)c2c(O)cccc21</smiles><smiles>CC(C)=CCCC(C)CCO</smiles><smiles>C=C(C)C(O)CCC(C)CCO</smiles><smiles>CC(C/C=C/C(C)(C)O)CCO</smiles>

Scheme 2. Example of photochemical reactions with sunlight.

added value of complexity-building photochemical steps should be factored in. ${ }^{[22,23]}$ In this spirit, the following example from our own laboratory shows the assembly of a bicyclic amino acid in a single step from readily available starting materials, by a photoinduced intramolecular para cycloaddition of an allene with an arene (Scheme 3). ${ }^{[24,25]}$

The inclusion of such steps is not limited to academic work in the sub-millimole scale, and industrial processes have taken advantage of photochemistry. One of the best known, and now quite old, processes is the synthesis of rose oxide (a fragrance ingredient) by Dragoco (now Symrise AG), using photogenerated singlet oxygen. ${ }^{[26]}$ But recently, a large-scale plant was dedicated by Sanofi to the synthesis of artemisinin, a potent anti-malarial drug. Noteworthy, liquid carbon dioxide was used as a solvent, thus limiting the generated wastes. ${ }^{[27,28]}$

The main efficiency observable in classical chemical transformations is undoubtedly the yield, reflecting how much of the reactant was actually converted into the product. Considering a photon as a reagent, one can extrapolate the corresponding yield with respect of photons, the quantum yield. And of course, all things being equal, maximizing the quantum yield ensures the most economic use of photons, which are energy-consuming to produce. On the other hand, one should keep in mind that the generation of photons is a relatively clean ('green') process, and thus if the increase in quantum yield is done at the expense of another less green parameter (e.g. by lowering the concentration, a typical move in photochemistry, leading to the use of large volumes of solvent), it may not be worthwhile.

The most appropriate green yardstick has been proposed to be the process mass intensity (PMI), defined as the total mass involved in a process, divided by the mass of the product that is being produced. ${ }^{[29]}$ As such, short synthetic routes involving a no-reagent photochemical step in as little solvent as possible is very likely to reduce the PMI, and thus to contribute to the greening of the production of fine chemicals. We mentioned earlier that maximizing yield at the expense of other parameters; one should not fall into the same trap being short-sighted and focusing only on PMI. The cost (financial and environmental) of setting up the facility, as well as satellite ingredients (catalysts, sensitizers) should be taken into consideration in the life cycle assessment. [30] Also, the strictly economic factor is only valid for a certain period of time, given the cost of energy and availability of certain resources.

In conclusion, we believe that photochemical reactions are indeed a valuable answer to most of the above-mentioned principles of green chemistry, provided that they are used in combination with other measures. Thus, principles 2, 6, 7, 8 and 9 are fulfilled by the very nature of photochemistry, whereas all the others, while not being directly addressed, are not incompatible with further improvements. In terms of engineering, many of the twelve principles are fully compatible with photochemistry, in particular with the very attractive possibility to adapt the reactions in flow, something that the solar photochemistry examples are ideally suited for. There is a bright future in photons!

Received: August 10, 2019

[1] G. Ciamician, Science 1912, 36, 385, DOI: 10.1126/science.36.926.385.

[2] D. G. Blackmond, A. Armstrong, V. Coombe, A. Wells, Angew. Chem. Int. Ed. 2007, 46, 3798, DOI: 10.1002/anie.200604952.

[3] Search on SciFinder on August 6, 2019 using Green photochemistry and Green chemistry as keywords and collecting answers as concepts. Similar results were obtained by using the keyword photochemical.

[4] B. M. Trost, Acc. Chem. Res. 2002, 35, 695, DOI: 10.1021/ar010068z.

[5] P. Anastas, J. C. Warner, in 'Green Chemistry: Theory and Practice', Oxford University Press: New York, 1998, p. 30.

[6] M. Poliakoff, P. Anastas, Nature 2001, 413, 257, DOI: 10.1038/35095133.

[7] S. Y. Tang, R. A. Bourne, R. L. Smith, M. Poliakoff, Green Chem. 2008, 10, 268, DOI: $10.1039 / \mathrm{b} 719469 \mathrm{~m}$.

[8] C. G. Bochet, Chimia 2016, 70, 182, DOI: 10.2533/chimia.2016.182.

[9] J. W. Tucker, C. R. J. Stephenson, J. Org. Chem. 2012, 77, 1617, DOI: 10.1021/jo202538x.

[10] C. Philippopoulos, D. Economou, C. Economou, J. Marangozis, Ind. Eng. Chem. Prod. Res. Dev. 1983, 22, 627, DOI: 10.1021/i300012a021.

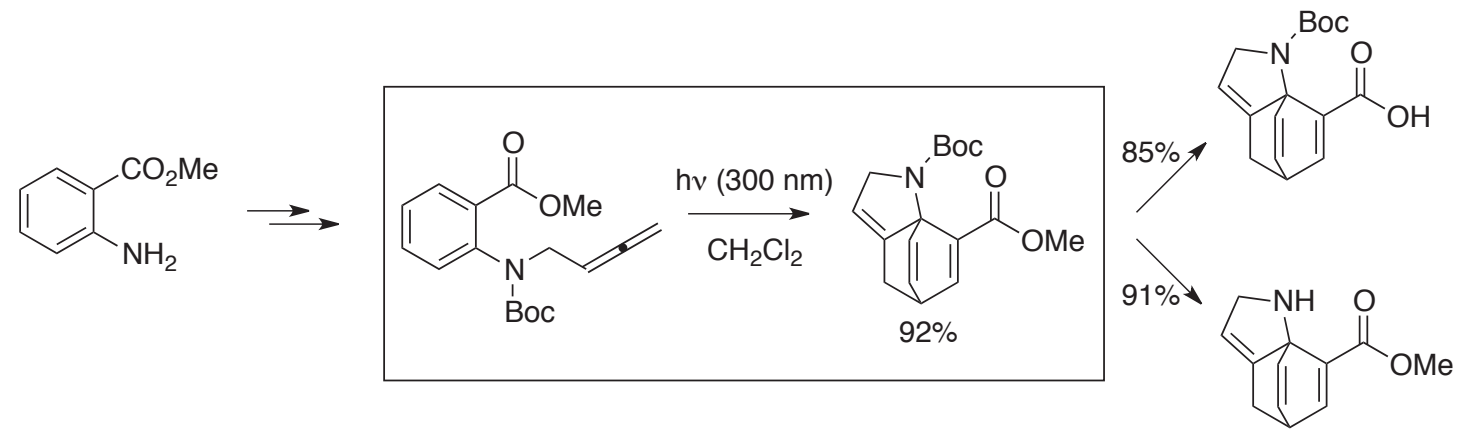


[11] S. Y. Reece, J. A. Hamel, K. Sung, T. D. Jarvi, A. J. Esswein, J. J. H. Pijpers, D. G. Nocera, Science 2011, 334, 645, DOI: 10.1126/science.1209816.

[12] M. G. Walter, E. L. Warren, J. R. McKone, S. W. Boettcher, Q. Mi, E. A. Santori, N. S. Lewis, Chem. Rev. 2010, 110, 6446, DOI: 10.1021/cr1002326.

[13] M. Oelgemöller, C. Jung, J. Ortner, J. Mattay, E. Zimmermann, Green Chem. 2005, 7, 35, DOI: 10.1039/b414230f.

[14] C. Schiel, M. Oelgemöller, J. Ortner, J. Mattay, Green Chem. 2001, 3, 224 DOI: $10.1039 / \mathrm{b} 106425 \mathrm{~h}$

[15] N. Charbonnet, PhD Thesis University of Fribourg, Fribourg, Switzerland, 2011

[16] E. J. Corey, M. Ohno, R. B. Mitra, P. A. Vatakencherry, J. Am. Chem. Soc. 1964, 86, 478, DOI: $10.1021 / \mathrm{ja} 01057 \mathrm{a} 039$.

[17] W. Oppolzer, T. Godel, J. Am. Chem. Soc. 1978, 100, 2583, DOI: 10.1021/ ja00476a071.

[18] G. Stork, F. H. Clarke, J. Am. Chem. Soc. 1955, 77, 1072, DOI: 10.1021/ ja01609a104.

[19] P. A. Wender, J. J. Howbert, J. Am. Chem. Soc. 1981, 103, 688, DOI: 10.1021/ja00393a041.

[20] P. A. Wender, J. J. Howbert, Tetrahedron Lett. 1982, 23, 3983, DOI: 10.1016/ S0040-4039(00)88675-X.

[21] G. Metha, A. N. Murthy, D. S. Reddy, A. V. Reddy, J. Am. Chem. Soc. 1986, 108, 3443, DOI: $10.1021 / \mathrm{ja} 00272 \mathrm{a} 046$
[22] M. D. Kärkäs, J. A. Porco, C. R. J. Stephenson, Chem. Rev. 2016, 116, 9683, DOI: 10.1021/acs.chemrev.5b00760. [23]

T. Newhouse, P. S. Baran, R. W. Hoffmann, Chem. Soc. Rev. 2009, 38, 3010, DOI: 10.1039/ b821200g.

[24] F. Birbaum, A. Neels, C. G. Bochet, Org. Lett. 2008, 10, 3175, DOI: 10.1021/ ol800806a.

[25] U. Streit, F. Birbaum, A. Quattropani, C. G. Bochet, J. Org. Chem. 2013, 78, 6890, DOI: $10.1021 /$ jo4002307.

[26] V. Nardello-Rataj, P. L. Alsters, J.-M. Aubry, in 'Liquid Phase Aerobic Oxidation Catalysis: Industrial Applications and Academic Perspectives', John Wiley \& Sons, Ltd, 2016, pp. 369, DOI: 10.1002/9783527690121. $\operatorname{ch} 22$.

[27] J. Turconi, F. Griolet, R. Guevel, G. Oddon, R. Villa, A. Geatti, M. Hvala, K. Rossen, R. Göller, A. Burgard, Org. Process Res. Dev. 2014, 18, 417, DOI: 10.1021/op4003196.

[28] Z. Amara, J. F. B. Bellamy, R. Horvath, S. J. Miller, A. Beeby, A. Burgard, K. Rossen, M. Poliakoff, M. W. George, Nature Chem. 2015, 7, 489, DOI: 10.1038/NCHEM.2261.

[29] C. Jimenez-Gonzalez, C. S. Ponder, Q. B. Broxterman, J. B. Manley, Org. Process Res. Dev. 2011, 15, 912, DOI: 10.1021/op200097d.

[30] C. K. Lee, H. H. Khoo, R. B. H. Tan, Org. Process Res. Dev. 2016, 20, 1937, DOI: 10.1021/acs.oprd.6b00275. 